\title{
A Grid Enabled Framework for Ubiquitous Healthcare Service Provisioning
}

\author{
Oludayo, O., Olugbara ${ }^{1}$, Sunday, O. Ojo², and Mathew, O. Adigun ${ }^{3}$ \\ ${ }^{1}$ Durban, University of Technology \\ ${ }^{2}$ Tshwane Univerity of Technology \\ 3 University of Zululand \\ South Africa
}

\section{Introduction}

The trend in modern healthcare is towards making quality services ubiquitous so as to improve accessibility and to reduce costs of services. The aspiration of government around the world is to improve the wellbeing of citizens and to deliver quality services that can add value to the people. Healthcare is one of those important services that can significantly improve the wellbeing of citizens and add value to people's quality of life because metaphorically, health is wealth. Moreover, healthcare represents a huge portion of Gross Domestic Products (GDP) and it sustains a strong public interest across the world. But, the conventional healthcare system is generally being confronted with the problems of low quality of services, lack of easy accessibility to service varieties and high costs of services.

The advancement in healthcare technology and the constantly changing nature of healthcare environment have brought about high costs of treatments. Many hospitals across the world cannot afford to pay for high costs of sophisticated healthcare equipment to provide costeffective services. Those few special hospitals that are able to afford the costs of expensive healthcare equipment are charging patients high service fees to breakeven. These healthcare equipment are available in specialized hospitals having highly skilled experts to utilize them successfully. This implies that people who are living in rural areas need to travel long distances to access quality healthcare services at expensive costs.

Moreover, disabled people and nomadic individuals living in rural seclusion find it difficult and expensive traveling long distances to access quality healthcare services. These challenges are particularly conspicuous in developing countries and rural world, making the realization of the health related Millennium Development Goals (MDG) a mirage. The general challenges facing the rural world are intermittent power, intermittent connectivity, long travel distances, variable population density, limited education, underemployment, limited disposable income and lack of secure storage (Parikh \& Lazowska, 2006).

Even in the developed world where there are claims of highly skilled expertise and accessibility to sophisticated healthcare resources, there is still the problem of aging population in this part of the world. The aging population quandary has led to more disabilities and is continually pulling renowned experts out of active services. This has serious ramifications on the social expenses incurred on healthcare. There is also evidence that low quality of service provisioning caused by variation and dichotomy in healthcare 
services and misuse of healthcare resources is also a challenge in many parts of the world. A comprehensive discussion on the general challenges facing the healthcare sector has been given (Sneha \& Varshney, 2006).

The process of healthcare management is indisputably a life-critical one that demands for the involvement of human beings to manage the health of others. Patients' health data are usually kept and managed securely within an hospital environment. Anywhere a patient goes, the history of his/her treatments is supposed to follow, but this is currently not the case around the world. Each time a patient visits a new hospital there would be demand for history of treatments from the patient. Consequently, patient mobility is not supported by the conventional healthcare system. This process definitely requires transformation in order to accurately diagnose a patient, because it is practically impossible for patients to correctly remember all the history of their treatments for accurate diagnosis to take place.

The non-standardization of diagnosis and treatment often expressed by the slogan different hospital, different treatment is another important issue in healthcare management. Moreover, the direct involvement of human beings in the main activities of healthcare implies high probability for making mistakes as a result of possible incorrect prescriptions of medications that can be made for patients and this can lead to lives being lost. An important question to ask is to what extent can healthcare practitioners avoid mistakes? It is difficult to completely avoid human mistakes, but mistakes can be considerably minimized by using automated procedures, tools and systems to support our daily activities and to transform the conventional ways that we do things. Automated systems have the capability to correct a user in the process of making mistakes.

The conventional healthcare system therefore, strives to overcome the difficulties inherent in the management of patient data, standardization of diverse data formats to support interoperability, acquisition of data from remote patients, efficient extraction and analysis of relevant content knowledge, federation of disparate healthcare databases as well as privacy and confidentiality of healthcare information. As such, many private and public hospitals are still in the practice of using conventional healthcare system to facilitate the process of diagnosis, treatment and prescription. This process is mainly manual, labor intensive, prone to mistakes and involves much paper work and the use of standalone systems for productivity enhancement. This approach gives variation and disparity in healthcare services and makes standardization and interoperability of healthcare infrastructure extremely difficult.

Today, the healthcare challenge facing many developing countries, including South Africa is how to extend quality and affordable healthcare services to a large number of people, especially those in the rural areas. This challenge suggests the necessity for ubiquitous healthcare service provisioning system and attests to the relevance of this study to our immediate society. The remainder of this chapter is succinctly outlined as follows: Section 2 presents our contributions. Section 3 discusses the state of the art in telemedicine technology. Section 4 discusses the state of the art in grid computing in healthcare. Section 5 presents our grid enabled framework for ubiquitous healthcare service provisioning. Section 6 presents an evaluation of our framework based on ubiquitous evaluation areas. Section 7 gives conclusion of the chapter and discusses our future work.

\section{Contributions}

This chapter describes our current effort to design and evaluate a grid enabled framework for ubiquitous healthcare service provisioning. The limitations of conventional healthcare 
system are low quality of service provisioning, lack of easy accessibility to service varieties and high costs of services. But, the emergence of technologies such as telemedicine, wireless body area network and wireless utility grid computing provides a unique opportunity to overcome the limitations of conventional healthcare system. These technologies can enable improved quality of healthcare by eliminating variation and dichotomy in healthcare services and misuse of healthcare resources. Moreover, they can provide easy accessibility to service varieties by allowing distributed healthcare resources to be massively reused to provide cost-effective services without individuals physically moving to the locations of those resources. In addition, they can reduce costs of healthcare services by allowing individuals to access ubiquitous services to support their healthcare.

Exploiting these novel technologies, our contribution is threefold. First we examine the roles of emerging technologies in enabling ubiquitous healthcare service provisioning. Second we illustrate how these technologies can be integrated to realize a grid enabled framework for ubiquitous healthcare service provisioning. Third we provide an evaluation of our framework based on ubiquitous computing evaluation areas. Moreover, our framework was compared with some three other existing ubiquitous healthcare frameworks and it promises to address the challenges of healthcare system better than those that it compares with by taking advantages of grid computing and mobile computing technologies. In future, we plan to deploy our framework and to test its efficacy for enabling ubiquitous rural healthcare service provisioning.

\section{Telemedicine technology - state of the art}

The significant roles of telemedicine technology in enabling high quality, cost-effective and easy accessibility to healthcare services are enormous. Telemedicine is the science and art of the maintenance of health, prevention, alleviation, rehabilitation and cure of diseases in patients using Information and Communication Technologies (ICT) (Van Beijnum et al., 2009). The main objective of telemedicine is to improve quality of healthcare and to minimize costs of healthcare services by making communication of crucial information between healthcare practitioners easy.

Telemedicine facilities include virtual reality, virtual community, mobile virtual community, mobile dynamic virtual community and wireless body area network and it potentially

a. Provides a means to effectively share knowledge and to overcome several challenges caused by geographical locations, scarcity of qualified healthcare practitioners and inadequate transportation systems to rural areas.

b. Brings healthcare services more closely to the people, improves quality and accessibility to healthcare services and minimizes number of case referrals.

c. Saves patients the inconvenience, stress and cost of travelling long distances to specialist centers for treatment because diagnosis and treatment can be provided at a distance.

d. Bridges the gap between highly curative healthcare services often concentrated in urban areas and pitiable healthcare services sometimes found in rural areas.

\subsection{Virtual Reality}

Virtual Reality (VR) as a telemedicine facility has attracted much research interest in healthcare. VR is a simulation of a real or an imagined environment that can be visually experienced in 3-dimensions and that may provide an interactive visual experience in real- 
time motion with sound and tactile feedback (Roy, 2003). The main characteristic of VR technology is the full immersion of human sensory-motor channels into a vivid and global communication experience (Riva \& Gamberrini, 2000; Roy, 2003). These authors asserted that VR based healthcare systems provide the following three important aspects to offer new possibilities for assessment and treatment of diseases. It allows for

a. Direct manipulation interfaces to allow content manipulation through pointing devices such as mouse and joystick. VR systems allow the manipulation of multiple sensory representations of entire environment by natural actions and gestures.

b. Effective control method to monitor, capture and process all properties of body actions or signals. These actions are then translated into other actions that have more effect on the world being controlled.

c. Capability to display feedbacks in multiple modalities. The feedbacks are then translated into alternative senses for users with sensory impairments.

VR based remote environments and related technologies provide improved healthcare services and savings in material resources (Moline, 1997). VR technologies have been applied to different healthcare applications including the augmented surgery and surgical training that are critically dependent on eye-hand coordination (Riva \& Gamberini, 2000) and the treatment of phobias (Roy, 2003). But, they are still faced with the problems of high costs of Reduced Instructions Set Computer (RISC) platforms upon which they are based, lack of reference standards and non-interoperability of the systems (Riva \& Gamberini, 2000). Moreover, VR worlds are generally believed to be visually unconvincing and full of encumbrances.

\subsection{Virtual Community}

Virtual Community (VC) based solutions to healthcare system allow for the building of shared communities, networks and organizations contexts of understanding and knowledge where the real-world knowledge affects the virtual interaction that modifies real-world therapies (Scarlet et al., 2007). A VC is a group of people who gather because of a common interest such as healthcare service provisioning and whose members interact independent of time and space using ICT as a platform (Leimeister et al., 2002). Scarlet et al. (2007) proposed a VC home rehabilitation approach as a realistic solution to the Romanian healthcare system. This allows for the possible rehabilitation of patients that are geographically dispersed and to access a distributed virtual platform that is able to provide communication and shared knowledge with healthcare practitioners (doctors, nurses and therapists), social workers and those involved in the rehabilitation process.

There are different criteria proposed in the literature for characterizing VC and examples include (Porter, 2004; Van Beijnum, 2009):

a. Purpose for the community to exist and shared common interests among community members.

b. Structural property of the community where the interaction happens, whether the community members interact in a physical location or the interaction is mediated by technologies whilst in different geographical locations.

c. Interaction platform or modality among the community members that is supported. This can be synchronous, asynchronous or hybrid of both interaction platforms.

d. VC defined three options of population, which are VC as computer supported social networks, VC as small groups and VC as networks. 
e. VC provides a profit model to know whether the community is revenue generating or non-revenue generating.

But, the challenges in VC research have been identified to include the need to (Abuelmaatti \& Rezgui, 2008):

a. Clarify and define the nature of virtual business modes that take place amongst communities.

b. Specify the technological, regulatory and socio-organizational environment to support communities effectively.

c. Discover the factors that facilitate virtual business modes adoption and use across communities.

\subsection{Mobile Virtual Community}

The wide proliferation of mobile wireless consumer devices, simply called mobile devices and the advancement in wireless positioning technologies for location based services have led to the emergence of Mobile Virtual Community (MVC) (El Morr \& Kawash 2007; van Beijnum et al., 2009; van't Klooster et al, 2010). A MVC can be considered as a natural evolution of a VC to which mobile services are added. A MVC facilitates real-time social connection and social interaction amongst community members anywhere anytime.

The important properties of a MVC that differentiate it from a VC are discussed as follows (Van Beijnum et al., 2009). First, MVC can make use of mobile service platform such as Short Message Service (SMS), mobile messaging and mobile Voice over Internet Protocol (VoIP) to support effective communication. Second, MVC offers enhanced mobile services that provide users with the ability to interact within the community anytime anywhere. Third, MVC results in entirely different usage patterns as it focuses on an individual user rather than around a specific interest. Apart from the fact that a MVC is a VC with extra mobility support, MVC can generally be characterized as follows (Rheingold, 2003):

a. VC, internet resources and their software agents are instantly available in MVC irrespective of their locations.

b. MVC is used to coordinate actions of groups of people in diverse geographic spaces.

c. MVC provides gaming environments and can start with young people as means for entertainment and light social interaction and then diffuse into other institution.

There are different dimensions to viewing mobility issues in $\mathrm{VC}$, but mobility is generally associated with the use of mobile devices to facilitate effective information communication and interaction. The following four basic modes of mobility were defined (Schulzrinne \& Wedlund, 2000; Van Beijnum et al., 2009):

a. Terminal mobility relates to the mobility of a device and it allows an end-device to be connected to the communication network while being on the move. Terminal mobility support will cause network connections to remain whenever there is a change between access points.

b. Session mobility allows a user changing from using one terminal to another to maintain the ongoing media session. This mobility is achieved by Session Initiation Protocol (SIP) to enable the transfer of application sessions between different devices without interruption.

c. Personal mobility allows a user having multiple terminals to be addressed by one and the same logical address. This form of mobility supports the user to change its device on-the-fly and the state is transferred from one device to the other, thereby allowing seamless terminal changes. 
d. Service mobility is the capability of using services in the case of moving or changing devices and network service providers. This form of mobility includes any relevant personal service configuration setting or preferences.

A MVC is a useful facility for providing interaction between patients, healthcare practitioners and hospitals. Patients need to share experiences and testimonies with one another for emotional support. They also participate in self-help group activities to obtain information and to establish interactions to assist in the prevention and treatment of diseases. But, according to El Morr \& Kawash (2007), little research has been conducted to understand how network providers can turn MVC services into profit making venture and to discover requirements and acceptance trends of users. Moreover, MVC is not likely to support strong community cohesion as community memberships can be short lived and communities can suffer from weak membership commitments. This is because a common interest does not necessarily lead to membership loyalty and durable cooperation.

\subsection{Mobile Dynamic Virtual Community}

A Mobile Dynamic Virtual Community (MDVC) is a VC whose members are able to change locations while the provided services remain available (Waldburger \& Stiller, 2005). MDVC extends $\mathrm{VC}$ with respect to dynamicity and mobility and service provisioning is concerned with the entity providing services. Akogrimo support for DMVC completely differentiates it from many other existing grid based solutions. The driving force behind the Akogrimo project is to merge large scale properties of telecommunication platforms and their support for multimedia applications with the cross-organization collaboration concepts of serviceoriented Grids into a coherent and integrated service platform (Jähnert, et al., 2010).

A MDVC is characterized by a strong dynamic element with respect to its organizational composition and business processes. The dynamicity and mobility characterization of MDVC is explained as follows (Waldburger \& Stiller, 2005):

a. Dynamicity, as members can freely join or leave the community and there is the need to manage entity that arises during the active existence of members in the community.

b. Mobility, if a Dynamic Virtual Community (DVC) allows at least for a subset of its members to change locations and still being able to profit or to provide services while moving, the DVC is called a MDVC.

\subsection{Wireless Body Area Network}

The Wireless Body Area Network (WBAN) (Van Dam, et al., 2001; Latre, et al., 2010) is an emerging wireless sensor networks telemedicine facility that uses intranet, internet or satellite communication for remote patient vital signs monitoring. WBAN technology allows for the automatic real-time acquisition of vital signs to provide flexibility and to support patient mobility. The technology is used in surgical and intensive care units of modern hospitals, at homes and in emergency situations to rescue patients who need urgent help. WBAN uses wearable sensor devices and actuators, mobile devices and wireless network devices to manage physical health conditions of patients. The health conditions are usually indicated through some physiological signals such as body temperature, body weight, waistline, blood pressure (BP), pulse rate (PR), oxygen saturation ( $\mathrm{SpO} 2)$, blood sugar electrocardiogram (ECG), electroencephalograph (EEG) and electromyography (EMG) without any particular emphasis on geographical locations of patients. 
The sensors and actuator devices, simply called motes can remotely collect many vital signs for diagnosing patients' health conditions and providing efficient and cost-effective treatment in real-time. A critical component of WBAN is a device Network Connector (NC) or hub that is capable of processing, displaying and transmitting sensors information to a remote telemedicine system. Because sensors do not need to communicate with each other, they communicate with a central Personal Server (PS) through the NC. The PS either processes the information sensed from human body and displays the results through a multimodal interface or transmits the sensed information to a telemedicine system.

ZigBee and the Bluetooth standards are generally used for radio interface between motes and PS in WBAN systems. However, performance, reliability and security of WBAN based healthcare applications have been investigated (Ylisaukko-oja et al., 2004; Li et al., 2008; Chavez-Santiago et al., 2009; Nam, 2009; Peiravi \& Farahi, 2010). Interestingly, the suitability of ZigBee and Bluetooth for healthcare applications has been questioned. ZigBee has high vulnerability to interference, Bluetooth has scalability problem and both ZigBee and Bluetooth support low data rate communication. They are therefore, not suitable for novel applications such as wireless medical imaging (Chavez-Santiago et al., 2009).

The Ultra WideBand (UWB) (Ryckaert et al., 2005) transmitter with low power consumption and low complexity was proposed to be used in the WBAN context. Chavez-Santiago et al. (2009) also proposed UWB for wireless interface between sensors and a PS because of the several advantages of the wireless technology. For examples, UWB signals do not cause significant interference to other systems operating in the vicinity. They do not constitute a threat to patient's security because they protect the transmission of patient's data by reducing the probability of detection. This therefore, rescinds the need for health data encryption that results in lower complexity of the electronics and smaller size of the devices. A novel Signal to Noise Ratio (SNR) estimator algorithm was designed for quality control in UWB communication (Barrera et al., 2010).

\section{Grid Computing in healthcare - state of the art}

El Morr \& Kawash (2007) asserted that ubiquity of MVC is a reconciliation factor to keep virtual communities going smoothly. They justified this assertion that while a common interest can keep a member attached to the community, this can only occur for a rather transient period of time, but that community hopping can be a sustaining power for MVC. Ubiquity of MVC can be facilitated by emerging technologies such as mobile web services and mobile grid computing by allowing distributed resources and expertise to be massively shared. A Grid is able to effectively manage different kinds of hardware and software resources that are within its environment. It can integrate web services, distributed hospital information system, high performance computing, interoperable health records and Peer-toPeer (P2P) system to provide electronic infrastructure. The real and specific problem that underlies grid concept is coordinated resource sharing and problem solving in dynamic, multi-institutional virtual communities (Foster et al., 2001).

Moreover, as demonstrated by Shaikh, et al. (2009) web services or more generally, Service Oriented Architecture (SOA) is known to play a significant role in telemedicine systems for solving interoperability problem. This then allows diverse telemedicine systems to effectively talk to each other. Grids are classified according to the functionalities they offered and the applications they supported. Commonly used grid taxonomy include computational grid, data grid, service grid, interaction grid, knowledge grid, utility grid, 
science grid, bio grid, sensor grid, cluster grid, Healthgrid, campus grid, Tera grid and commodity grid. However, the following checklist has been proposed to determine the real meaning of a grid system (Foster, 2002): a grid is a system that

a. Coordinates resources that are not subject to a centralized control and it addresses the issues of security, policy, payment and membership that arise in this setting.

b. Uses general purpose protocols and interfaces that are standard and open to address issues such as authentication, authorization, resource discovery and access to resources.

c. Delivers nontrivial quality of service relating to response time, throughput, availability, security and co-allocation of multiple resources to meet complex user demands.

Examples of application demonstrations of the usefulness of grid computing to healthcare include Bioprofiling over the grid for healthcare in which BIOPATTERN grid was developed for Biopattern analysis and Bioprofiling to support individualization of healthcare (Sun et al., 2006). The BIOPATTERN grid was designed to facilitate secure and seamless sharing of geographically distributed Bioprofile databases and to support the analysis of Bioprofiles to overcome diseases such as brain and cancer. The distributed mammography data retrieval and processing (MammoGrid) (Amendolia et al., 2005), the eDiaMoND (Lioyd et al., 2005) and the multi-centre neuro-imaging (BIRN) (Grethe et al., 2005) are also examples of useful applications of grid computing to healthcare.

Additionally, the Intensive Care Grid (ICGrid) (Gjermundrod et al., 2007) is a novel system framework based on the Enabling Grids for E-sciencE (EGEE) to enable the seamless integration, correlation and retrieval of clinical interesting episodes across Intensive Care Unit (ICUs). The ICGrid allows doctors to utilize the EGEE grid infrastructure through simple intuitive user interfaces, while the infrastructure itself handles the complex task of information replication, fault tolerance and sharing (Gjermundrod et al., 2007). The CardioGrid (Risk et al., 2009) is a grid based framework for analyzing cardiological signals. This infrastructure allows a user to process on the grid, the electrocardiogram (ECG) signals obtained from a portable data acquisition device. The World-wide In Silico Docking On Malaria (WISDOM) is a European initiative to enable the in Silico drug discovery pipeline on a grid infrastructure. The WISDOM initiative demonstrated an efficient process of drug discovery by speeding up the entire process and reducing the cost to develop new drugs to treat diseases such as malaria (Jacq et al., 2006).

\subsection{Grid computing technology}

A grid is a flexible distributed information technology environment that enables multiple services to be created with a significant degree of independence from the specific attributes of underlying support infrastructure (Travostino et al., 2006). Grid computing is conventionally associated with high computational capability and ability to handle enormous amount of data to be processed (Foster \& Kesselman, 1999; Berman et al., 2003). Grid provides a means for massively distributed parallel processing such as Search for Extra-Terrestrial Intelligence (SETI), Symmetric Cryptographic Cracking (SCC), Human Genome Project (HGP), MOlecular Visualization (MOV) and Multimedia Content Distribution (MCD). Grids are determined by the following three main characteristics: resources coordination, general purpose protocol and quality of service support (Foster, 2002). The pervasive nature of grid allows remote computing resources to be accessible from different platforms (desktops, laptops, PDAs, mobile phones) using the web browser. The Healthgrid community is focusing on the use of grid in healthcare management as a way to resolve the challenges of conventional healthcare system. 
Grid middleware infrastructures such as Condor, Gridbus, Globus and gLite provide functionalities for security (authentication and authorization), resource management (resource allocation and job management), data management (grid file transfer protocol and replica management) and data service support such as Grid Service Architecture Data Access and Integration (OGSADAI). The Condor is an open-source grid middleware that allows users to reliably submit jobs to grids. The Gridbus aims to develop next-generation cluster and grid technologies that support utility-based service-oriented computing. The Globus is an open-source codebases and a set of protocols for implementing grid applications. The gLite is a lightweight middleware framework of EGEE for building grid applications.

\subsection{Wireless Grid Computing}

A wireless grid is a virtual network system that integrates different types of wireless devices, thereby extending grid computing capability to wireless devices such as sensors, actuators, mobile phones, laptops, special instruments and edge devices. Wireless Grid Computing (WGC) supports the sharing of grid resources amongst mobile and fixed wireless devices within a VC. The general characteristics of WGC are summarized as follows (Manvi \& Birje, 2010): It

a. Does not provide centralized control of resources.

b. Consists of small and low powered devices.

c. Includes heterogeneous resources, applications and interfaces.

d. Allows sharing of new types of resources types like cameras, GPS trackers and sensors.

e. Is dynamic with unstable users and resources.

f. Provides geographically dispersed resources with different management policies.

g. Provides different security requirements and policies.

Wireless grids can be classified according to their relative accessibility and interaction modality supported. Four categories of wireless grids are (Li et al., 2009; Manvi \& Birje, 2010):

a. Fixed wireless grids extend grid resources to wireless devices of varying sizes and capabilities where these devices are usually static. In this type of grids, wireless devices can act as real nodes for data processing and storage to take place.

b. Mobile wireless grids make grid services accessible through mobile devices such as smart phones and Personal Digital Assistants (PDA). This type of grids allows devices to connect to the internet, support P2P network connection, take advantage of the resources of wired grid networks and make their own resources available to the wired grids.

c. Mobile Ad Hoc grids consist of devices with a high degree of heterogeneity. These grids can be instantly constructed anytime, anywhere, especially for an emergency situation by integrating grid resource aggregation model with mobile ad hoc networks.

d. Sensor network grids are composed of tiny devices dedicated to a single purpose and they integrate detection, processing and communication capabilities into grids.

Due to mobility of wireless devices and inherent limitations of communications technology, a number of challenges have to be surmounted to build robust grid applications for resource constrained devices. This is because wireless devices were not viewed as having sufficient capability to be both clients and services (Chu \& Humphrey, 2004). Some of the 
requirements and challenges of wireless grids have been discussed (Manvi \& Birje, 2010). Among those research efforts that currently extended mobility into grids to address resource limitations and intermittent network connectivity problems associated with wireless devices are:

a. Mobile Open Grid Service Infrastructure (OGSI) dot net (OGSI.Net) (Chu \& Humphrey, 2004) is an important step towards making mobile devices first-class entities in grids based on OGSI or Web Service Resources Framework (WSRF). OGSI extends the statelessness of web services to accommodate grid computing resources that are transient and stateful. WSRF captures the state requirement of web services, making it possible to use grid services as web services. Both OGSI and WSRF provide a unique support to host grid services on mobile devices, but WSRF seems more promising with the release of Globus toolkit 4 (Foster, 2006), which implemented WSRF.

b. Wireless Grid based Mobile Agent System (WGMAS) (Leu \& Wang, 2006) is a grid service platform that integrates mobile agent technique and wireless environment with a grid system to provide a wireless access mobile agent system to users.

\subsection{Utility Grid Computing}

Grid computing was originally introduced as a means to solve high computationally intensive scientific problems. But, the influence of grid computing has speedily extended from scientific applications to more resourceful applications domains such as healthcare, business and commerce. Utility Grid Computing (UGC) extends the original grid computing to incorporate business model and it presents resource management as a crucial element of grid computing. The utility model of grid computing draws on an analogy to the commercial electrical power system for metered service provisioning.

The electrical power from any generating, transmitting and distributing stations can serve the purpose of any consumer without a geographical obstacle. Moreover, consumers of electrical power do not need to worry about the complicated intricacies of the electrical transformers and the peak load management that occurs through the power generation, transmission and distribution grids. This implies that accessing computer power from a computer grid would be as simple as accessing electrical power from an electrical grid and one would be able to request for computing services and get it on payment. The Harvard Presentation (HP) grid Utility Data Center (HP/UDC) that focused on the data center market and the International Business Machine (IBM) autonomic grid computing and E.Liza to build self-healing and self-organizing devices are two famous models of UGC.

\subsection{Wireless Utility Grid Computing}

Wireless devices are undoubtedly popular and generally accepted by everyone because of their impact, portability, low-cost and easy handling. The number of potential owners and users of wireless devices continue to amazingly increase on a daily basis, making these devices resourceful for boosting business opportunities. The amazing growth in the number of wireless device owners and users can be exploited to leverage numerous business opportunities, especially when wireless technology is fully integrated into grids. Wireless Utility Grid Computing (WUGC) extends the capability of UGC to support wireless technology. WUGC is rich in functionalities, can provide on demand access to appropriate services and can give high-bandwidth to a large constellation of distributed time-varying resources, thereby increasing the quality of service provisioning. 
Integrating wireless devices into utility grids to form WUGC provides an opportunity for sustainable ubiquitous healthcare service provisioning by harnessing the potentials of diverse resources on the grid. This will also create enormous new business opportunities as WUGC promises to deliver high quality services at low costs to alleviate the suffering of common people and to make small businesses to flourish. WUGC will definitely create enormous potentials for emerging resources such as cameras, microphones, videos, sensors and positioning system to improve ubiquitous healthcare service provisioning.

\subsection{Healthgrid and Grid Service discovery}

Healthgrid is an emerging grid based technology that promises to resolve a number of complicated healthcare challenges by integrating healthcare information systems, practitioners and diverse resources into a common, usable, accessible and shareable platform. Healthgrid provides an environment where healthcare data are stored, processed and made available to end-users as on demand services. Several Healthgrid projects are currently being undertaken to facilitate health data systems interoperability and to support improved data management, diagnosis and treatment of diseases. Examples of such projects include DataGrid (Breton et al., 2003), e-Diamond (Brady et al., 2003), NeuroGrid (Geddes et al., 2005) and MEDIGrid (Boccia et al., 2005).

Grid service discovery is one of the most important functionalities expected from the Healthgrid and it generally represents an interesting problem in grid research. Grid service discovery is the task of matching a query for services described in terms of the required characteristics to a set of services that meet the expressed requirements (Kaur \& Sengupta, 2007). Grid service discovery is a kind of Constraint Satisfiability Problem (CSP) (Yokoo et al., 1998), complicated by the dynamic changes in service information. The challenge is to devise highly distributed service discovery techniques that are fault tolerant and highly scalable (Kaur \& Sengupta, 2007).

A Grid Service (GS) is a resource on the grid that is transparent to consumers and extends the stateless nature of a Web Service (WS) to deal with state and notification. A WS based grid extends the capabilities of WS by providing common and powerful mechanisms for service consumers to access all kinds of services. The Universal Description, Discovery and Integration (UDDI) plus rich query (Kaur \& Sengupta, 2007) is a novel web-services based grid services discovery model. UDDI already contains basic entities such as businessEntity, businessService, tModel and bindingTemplate for efficient service discovery when a powerful query model is applied. In a recent development, Naseer \& Stergioulas (2010) proposed a new novel WS based service discovery model for service discovery in Healthgrid based on WS and WS technologies to transform virtual healthcare into a reality.

\section{Grid enabled healthcare service provisioning}

The challenges facing the conventional healthcare system demand for improved infrastructure and novel methods for healthcare service provisioning. A Grid enabled Framework (gFrame) is proposed for ubiquitous healthcare service provisioning. The goal of gFrame is to integrate MDVC of healthcare service providers, individuals, personal space and smart space into Healthgrid for the purpose of enabling ubiquitous, quality and cost-effective healthcare service provisioning, thereby surmounting the challenges of the conventional healthcare system. In this context, services represent resources (experts, equipments and operations) that can be provided on the grid and consumed as web or grid services. 
Zhang et al. (2004) defined a personal space as a space equipped with a network of WBAN and enriched with context information, ranging from static information (particulars, contact number, subscribed health insurance and health profile) to dynamic information (health status, location and socializing record). Similarly, they defined a smart space as a physical environment (house, hospital and car) of individuals, whereby the context information is made available through sensors and actuators techniques and information technologies. Context information range from low-level context, such as temperature, noise level and location of coordinates to high-level context, such as activity schedule, relationship between individuals and event profile.

The integration of a MDVC into grids generally offers an enticing surrogate for resource demanding applications by leveraging the power of several devices to collectively provide the Quality of Service (QoS) required by individual service consumers. Our own specific reason for considering the integration of a MDVC of healthcare into Healthgrid is to help individuals in the rural world to access quality healthcare services at reduced costs within and across boundaries without the individuals moving to the physical locations that those services are produced. To describe the gFrame architecture, it is important to first discuss the services that can be provided within a typical VC of healthcare and on the Healthgrid and the challenges of integrating mobile devices into grids for enabling ubiquitous healthcare service provisioning.

\subsection{Virtual Community and Healthgrid services}

The healthcare services to be provided within a VC and on the Healthgrid have been discussed (van Beijnum et al., 2009; Naseer \& Stergioulas, 2010; van't Klooster et al., 2010) and these services were categorized into management-level and operational-level services (Naseer \& Stergioulas, 2010). The management-level services are responsible for making resources available within the community.

The operational-level services are used for monitoring, diagnosing and treating patients for illness. These services implement all types of operations that are related to healthcare service provisioning including treatment, follow-up, consultation, dose computation, self-care, mobile-care, preventive-care, home-care, clinical annotation, drug prescription, group based analysis and diagnosis.

\subsection{Integration challenges}

The era of ubiquitous computing provides interaction convenience and mobility support as individuals can now access services on the move and on the bed through mobile devices that they carry about. While mobile computing community has succeeded in entering virtually all application domains to enrich human lives with increased personal and business productivity, it has not yet entered into the grid computing domain. The ongoing debate amongst members of the grid community is whether mobile devices should be considered as clients into the grid that is originally meant for high performance computing. This argument is anchored on the lucidity of considering resource-constrained devices on the grid, especially when only a small fraction of internet-connected desktop computers contribute to the grid (Phan et al., 2002).

However, the fact that mobile devices represent a large percentage of the world computing power is sufficient for it to be leveraged for finding more ways to harness the enormous potentials. But, the limiting force in leveraging the enormous potentials of mobile devices in 
a grid enabled environment include, slower processing power, unreliable low bandwidth wireless connectivity, and unreliable extended period of complete disconnection, heightened power-consumption sensitivity, small secondary storage and lack of adequate security mechanism. Moreover, while wired resourceful devices such as desktop computers are designed to function continuously over a long period of time, mobile devices have shorter battery lifespan and the battery cannot automatically self-charge when the devices are engaged.

Several researchers have proposed a proxy-based architecture to address the logjams of integrating mobile devices into grids. This architecture plays two significant roles in the integration of mobile devices into grids. First is to execute a request on behalf of mobile devices using appropriate grid middleware system. The proxy system sends the result back to the requester, thereby shielding the service requester from device heterogeneity. In this particular scenario, a mobile device is regarded as a pure service consumer, thereby leading to a Client and Server (CaS) kind of architecture. Second is to distribute a request to be naturally executed on a single mobile device amongst several computing devices. The proxy system waits for the results and sends them to the requester. The second scenario seems to be more promising as mobile devices serve as both service consumers and service providers, thereby leading to a Peer-to-Peer (P2P) kind of architecture. But the data partitioning to be solved in P2P architecture is a challenging task within the parallel computing community. The proxy-based system provides an efficient way to integrate virtual communities into grids, instead of direct connectivity that does not solve many of the logjams of constrained devices. Table 1 shows some proxy-based architectures for integrating mobile devices into grids and the problems addressed.

\subsection{Framework description}

This section describes gFrame, which improves on our previous grid based Healthcare Service Broker (HSB) for remote vital signs management (Olugbara et al., 2007a, Olugbara et al., 2007b). This new framework extends HSB architecture to MDVC and it supports context awareness and personalized grid service discovery based on UDDI Technical Metadata $\left(\right.$ UDDI-MT) $^{\mathrm{T}}$ (Miles et al., 2003). The UDDI-MT approach supports the storage of arbitrary metadata through a tunneling technique that associates the metadata store to the original UDDI directory. Service metadata such as reliability metrics and QoS data can originate from both service providers and service consumers. The metadata registry can be queried using Resource Description Query Language (RDQL), which is a graph-based query language that exploits the graph structure of Resource Description Framework (RDF).

The design objectives of our gFrame that eventually translate into system requirements are the following.

a. To construct a MDVC infrastructure to facilitate collaboration amongst healthcare service providers and service consumers for the purpose of ubiquitous healthcare service provisioning.

b. To make the infrastructural framework operate on multiple device platforms to obtain a platform independent system configuration.

c. To address the particular characteristics of mobile devices in the framework using grid computing technology.

d. To support personal health, spatial-temporal and technical context awareness to allow healthcare service provisioning to be inherently personalized and mobile. 
e. To allow for the creation of local healthcare communities of patient categorise.

f. To allow patients to be grouped by their diseases to make easy for market volume estimation.

\begin{tabular}{|c|c|}
\hline Platform Description & Problems Addressed \\
\hline $\begin{array}{l}\text { Proxy-based clustered architecture (Phan et al., 2002) creates } \\
\text { clusters of baseline units with each cluster centered on an } \\
\text { interlocutor proxy, which represents minions to the grid. The } \\
\text { interlocutor runs appropriate grid middleware to publish itself } \\
\text { as a node that can contribute a certain amount of computational, } \\
\text { networking and storage resources. }\end{array}$ & $\begin{array}{l}\text { Device heterogeneity, } \\
\text { low-bandwidth, } \\
\text { high-latency } \\
\text { connectivity and } \\
\text { power consumption. }\end{array}$ \\
\hline $\begin{array}{l}\text { iMobile EE (Chen et al., 2003) is an enterprise mobile service } \\
\text { platform that provides a scalable, secure and modular software } \\
\text { platform, which makes enterprise services easily accessible to a } \\
\text { growing list of mobile devices. }\end{array}$ & $\begin{array}{l}\text { Security, scalability } \\
\text { and availability. }\end{array}$ \\
\hline $\begin{array}{l}\text { Mobile OGSI.NET (Chu \& Humphrey, 2004) promotes resource } \\
\text { sharing and collaboration that improves the user experience. It } \\
\text { extends an implementation of grid computing OGSI.NET to } \\
\text { mobile devices by adhering to the OGSI specification to support } \\
\text { the hosting of grid services on mobile devices. }\end{array}$ & $\begin{array}{l}\text { Resource limitations } \\
\text { and intermittent } \\
\text { network connectivity. }\end{array}$ \\
\hline $\begin{array}{l}\text { Scalable inter-grid network adaptation layers (Hwang \& } \\
\text { Aravamudham, 2004) integrates mobile devices with grid } \\
\text { platforms to conduct P2P operations through a proxy-based } \\
\text { middleware architecture that executes jobs submitted to mobile } \\
\text { devices. The proxy monitors other mobile nodes, providing } \\
\text { measurements, reporting actual QoS delivery and enforcing the } \\
\text { necessary resource reservations. }\end{array}$ & $\begin{array}{l}\text { Network and QoS } \\
\text { adaptation between } \\
\text { wireless devices and } \\
\text { network } \\
\text { infrastructure. }\end{array}$ \\
\hline $\begin{array}{l}\text { Lightweight grid platform (Isaiadis \& Getov, 2005) groups the } \\
\text { same subnet mobile devices to create a virtual cluster to be } \\
\text { presented to grid. The design uses a number of proxies that } \\
\text { provide the interface point between the grid and the lightweight } \\
\text { cluster of devices. }\end{array}$ & $\begin{array}{l}\text { Service availability, } \\
\text { failure recovery and } \\
\text { performance } \\
\text { enhancement. }\end{array}$ \\
\hline $\begin{array}{l}\text { Wireless grid-based mobile agent system (Leu \& Wang, 2006) is } \\
\text { a service platform that integrates agents, services, brokers and } \\
\text { resources to realize a robust and reliable environment. It is a } \\
\text { hard-wired grid integrated with wireless network and a SIP to } \\
\text { establish a wireless grid environment for user to access grid } \\
\text { resources through wired or wireless networks. }\end{array}$ & $\begin{array}{l}\text { Communication } \\
\text { failure, message } \\
\text { chasing and } \\
\text { maximizing resource } \\
\text { utilization. }\end{array}$ \\
\hline $\begin{array}{l}\text { Mobile service platform (van Halteren \& Pawar, 2006). is a } \\
\text { middleware based on JINI surrogate architecture to extend SOA } \\
\text { paradigm to mobile devices. It facilitates the development and } \\
\text { deployment of services on the mobile device for clients to } \\
\text { discover anywhere on the internet. }\end{array}$ & $\begin{array}{l}\text { Nomadic service } \\
\text { provisioning. }\end{array}$ \\
\hline
\end{tabular}

Table 1. Proxy-based architectures integrating mobile devices into grids 


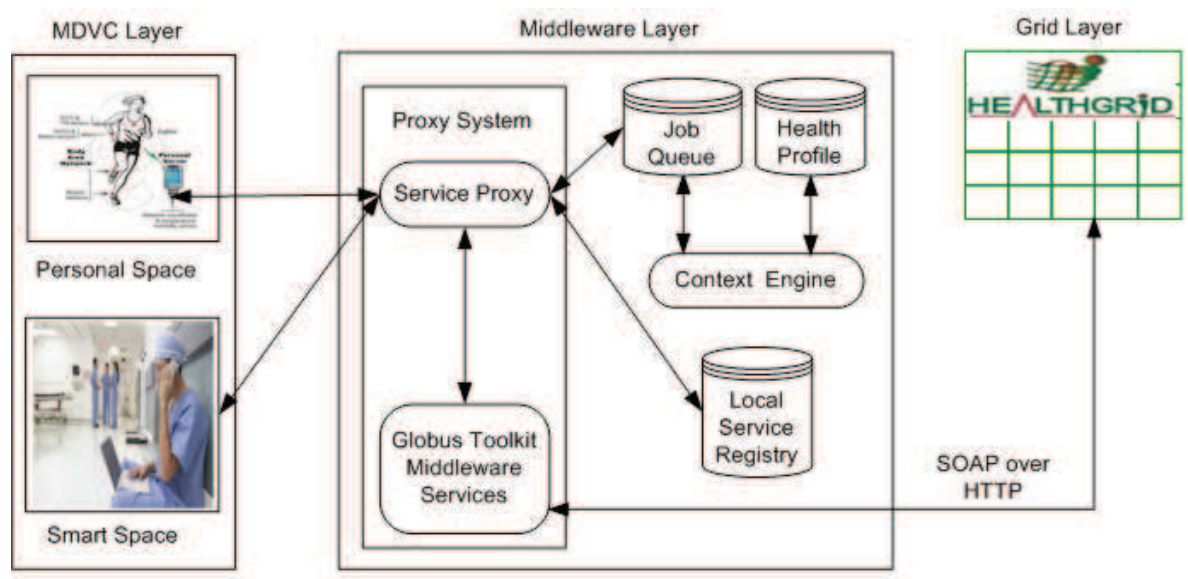

Fig. 1. The gFrame architecture

Figure 1 shows our gFrame architecture that features three main layers, MDVC, Middleware and Grid. The MDVC layer consists of personal space and smart space that interact with the middleware layer through wired/wireless communication network. The personal space provides access to motes that collect vital signs and environmental parameters in a Clinical Document Architecture (CDA) format. The smart space allows individual members of the MDVC to access community and Healthgrid services. The middleware layer integrates the MDVC to the Healthgrid through the Globus system using Simple Object Access Protocol (SOAP) over Hypertext Transfer Protocol (HTTP) as an ideal solution to access grid services. The Grid layer provides high quality services and supports the federation of diverse healthcare resources to be globally shared.

The middleware layer has proxy system, context engine, Local Service Registry (LSR) Job queue and Health profile database as components. The proxy system has a service proxy component that receives requests from the MDVC and it runs a Globus middleware to publish itself as a node that contributes to networking and service provisioning. The proxy system manages service requests from the MDVC using job queuing approach to achieve processing efficiency. Additionally, the proxy system is responsible for local and global service discovery within and outside the MDVC, respectively.

A local service discovery technique provides the opportunity for the global world to access local services that are provided within the MDVC. Similarly, the global service discovery enables the MDVC to benefit from some high-quality and cost-effective services that are offered outside the MDVC. The service proxy publishes certain frequently demanded grid services into the LSR to improve the efficacy of the service discovery algorithm. Whenever a particular service in the LSR is no longer visible or accessible the service proxy removes it from the LSR, thereby providing efficient service management functionality. The service proxy queues job and context requests into the job queue to be processed by the context engine and the service proxy is left with the tasks of service discovery and service rendition to the requester.

The context engine processes a context request and performs reasoning based on health and environment contexts associated with a request. The context engine is also responsible for generating patient-based recommendation services, determining when to generate 
specialist-based notification services about the current state of a client, managing patients and community-related profiles and generating adaptation services according to terminal context. In addition, the context engine acquires the context information from the job queue to produce a grid service based metadata request by performing an automatic service annotation in an appropriate format suitable for personalized service discovery. A Fuzzy logic system is proposed for the implementation of the context engine to be enriched with intelligence and explanation capabilities as well as ability to properly describe human reasoning. This enables the context engine to be able to provide an explanatory service for every decision taking on behalf of the service requester.

Our gFrame architecture integrates a MDVC of healthcare into the Healthgrid to potentially address the following research challenges.

a. Healthcare system challenges are addressed using an integrated MDVC and Healthgrid as a potential solution to provide ubiquitous, quality and cost-effective healthcare services.

b. Bandwidth availability and extended periods of disconnection are addressed, because grid applications naturally address these challenges and they do not depend on low latency in communication.

c. Device heterogeneity problem is potentially addressed using a proxy-based system to shield service requesters from the integration intricacies by making available the aggregated resources on demand.

d. Service discovery problem is simplified through personalized metadata, powerful query technique, web services and middleware technologies.

e. Power consumption problem is mitigated as much function will be performed by the proxies (service proxy and Globus system) on behalf of the service requesters.

\section{Framework evaluation}

This section considers evaluating gFrame based on Ubiquitous Evaluation Areas (UEA) (Scholtz \& Cansalvo, 2004). The purpose is to obtain a usable implementation of the framework through cross comparison with some existing ubiquitous healthcare system frameworks. The need to consider evaluating a design framework is important for revealing direct impacts of a system and framework evaluation offers the following technology and business gains (Kobryn, \& Sibbald, 2004; Scholtz \& Cansalvo, 2004; Shirazi \& Zahedian, 2009). To

a. Ensure conformance to architecture best practices and promote systems interoperability.

b. Help developers to become aware of strong and weak points of the system and ensure that the right business system is being developed.

c. Effectively reduce time, cost, energy and probability of system failure and to accelerate time to market.

d. Help stakeholders to get a uniform view of the system, support appropriate view of various stakeholders and to capture and protect intellectual property.

e. Provide a situation in which the system can be uniformly generated, developed and implemented by separating business logic from technical infrastructure.

f. Increase the knowledge of developers, generate better systems and reveal hidden properties of the systems through evaluation results. 
g. Improve comparability across research efforts and give a clearer picture of the final system to implement.

h. Enable the development of a more comprehensive structure and avoid overlooking of the ambivalent areas of system requirements.

i. Reduce defeat density and volatility rate that can be naturally caused by requirements unpredictability and ambiguity in system requirements.

\subsection{Evaluation metrics}

There are different methods and techniques for evaluating framework of architectures and architectural framework evaluation was considered crucial in order to develop information systems (Shirazi \& Zahedian, 2009). These authors developed a uniform method for evaluating the Department of Defense Architecture Framework (DoDAF) complaint products using a checklist created for the evaluation. However, the ubiquitous computing environment has more stringent and constrained usability requirements that make most of the evaluation techniques less effective. As a result, the UEA technique was specifically developed for evaluating ubiquitous computing application frameworks.

The UEA (Scholtz \& Cansalvo, 2004) has a set of nine evaluation areas with thirty five associated metrics and several conceptual measures. A metric associates meaning to a value by applying human quantitative judgment. These observable values when associated with meanings are called conceptual measures. However, not all of these areas, metrics and conceptual measures are directly applicable to all situations. A user has to decide on which of these areas, metrics and conceptual measures are applicable to the situation being considered. In our particular situation, only four UEA and ten metrics are applicable and the areas are trust, adoption, impact and invisibility.

Trust is the belief that a system will use the personal data it collects from patients appropriately and in innocuous manner. Adoption refers to willingness to use a system and the rate of use. Impact is the utility that the system offers for users and the contributions it makes to someone's life. Invisibility concerns the integration of the system into user environment to support the individual users. Table 2 shows the selected UEA metrics,

\begin{tabular}{|l|l|l|l|}
\hline UEA & Metric & Code & Conceptual Measures \\
\hline \multirow{4}{*}{ Trust } & Awareness & (T1) & $\begin{array}{l}\text { Ease of coordination with users in multi-user } \\
\text { application. }\end{array}$ \\
\cline { 2 - 4 } & Privacy & (T2) & Availability of user's information to others. \\
\cline { 2 - 4 } Adoption & Control & (T3) & Ability of users to establish right over their data. \\
\cline { 2 - 4 } & Value & (A1) & Perceived costs and benefits of the system. \\
\hline \multirow{4}{*}{ Impact } & Cost & (A2) & $\begin{array}{l}\text { Ease of acquiring, setting up and maintaining the } \\
\text { system. }\end{array}$ \\
\cline { 2 - 4 } & $\begin{array}{l}\text { Social } \\
\text { Acceptance }\end{array}$ & (M1) & System ability to attract large number of users. \\
\cline { 2 - 4 } & $\begin{array}{l}\text { Environment } \\
\text { Change }\end{array}$ & $\begin{array}{l}\text { Needs on users outside of norms to accept the } \\
\text { system. }\end{array}$ \\
\hline \multirow{2}{*}{ Invisibility } & Intelligibility & $\begin{array}{l}\text { Willingness to accommodate system in user's } \\
\text { environment. }\end{array}$ \\
\cline { 2 - 4 } & Customization & (I2) & User understanding of the system explanation. \\
\hline
\end{tabular}

Table 2. UEA based vehicle metrics for comparing system frameworks 
which we called vehicle metrics, and their corresponding codes and conceptual measures. The vehicle metrics provide a means to describe some outcomes, which we called tenor metrics. These tenor metrics are the particular outcomes that we expect the framework to deliver and they form the basic metrics for the frameworks evaluation. For example, we want the frameworks to address the three challenges of conventional healthcare system.

The purpose of the evaluation is to compare our gFrame with some existing ubiquitous healthcare system frameworks to determine its strengths and weaknesses. In doing so, we need to map the vehicle metrics onto the tenor metrics, which from the challenges of healthcare system are access to services, quality services and cost reduction. Table 3 shows our tenor metrics and their corresponding codes and conceptual measures derived from the following important healthcare considerations.

a. How the framework can help to improve accessibility to healthcare services.

b. How the framework can help to improve the quality of healthcare service provisioning.

c. How the framework can help to reduce the cost of healthcare service provisioning.

\begin{tabular}{|l|c|l|}
\hline Consideration & Code & Conceptual Measures \\
\hline Access to Services & A & Mobility support, community membership, quality of services. \\
\hline Quality Services & Q & Personalized service, service discovery, security and trust. \\
\hline Cost Reduction & C & Membership support, available aid and mobility support. \\
\hline
\end{tabular}

Table 3. Tenor metrics derived from healthcare considerations

The access to healthcare services can be directly influenced by quality of services, mobility support, collaboration and information sharing amongst community members. The quality of services can be influenced by the functionalities delivered and performance requirements. These may include personalized service functionality, service discovery, security and trust requirements. The costs of healthcare services can be reduced when there are membership support, available aid and mobility support. Moreover, providing more efficient utilization of healthcare resources, reducing number of case referrals and frequency of hospital visits can contribute to reduced costs of healthcare services. Figure 2 shows the mapping of the vehicle metrics onto the tenor metrics by associating their corresponding conceptual measures, making the tenor metrics a concrete choice for the evaluation.

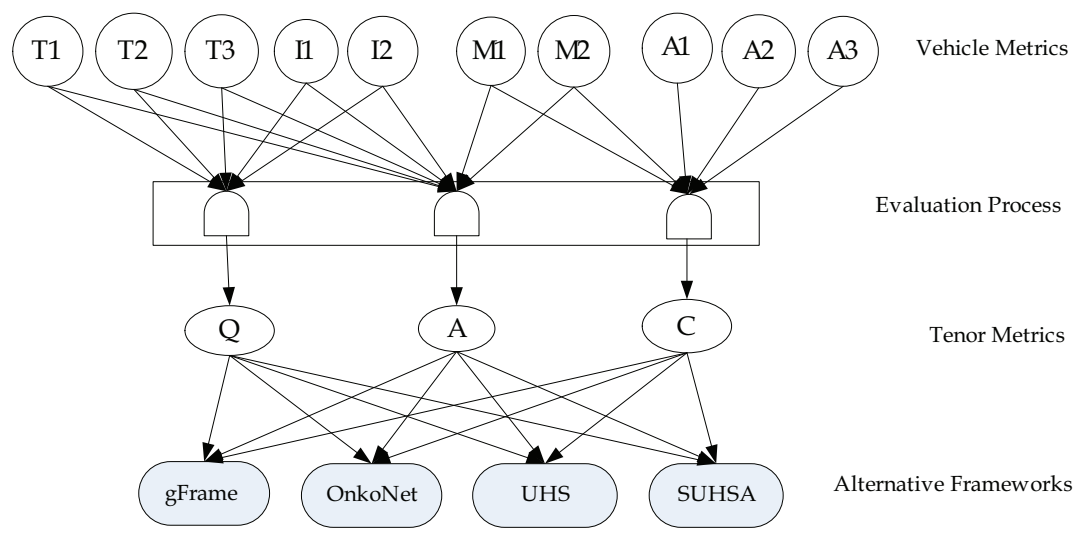

Fig. 2. Mapping of vehicle metrics onto tenor metrics for frameworks evaluation 
The mapping of the vehicle metrics onto the tenor metrics is described as follows. Quality of service is measured in terms of five vehicle metrics, awareness, privacy, control, intelligibility and customization. These metrics can guarantee that services are of high quality when the infrastructure supporting healthcare services can enable secure and ubiquitous discovery of personalized services, thereby satisfying the quality requirement of the tenor metrics. Cost reduction is measured in terms of the vehicle metrics, value, cost and availability. Based on cost reduction tenor metrics, membership support, availability of aid and mobility support can guarantee reduced costs of healthcare services. Access to services is measured in terms of vehicle metrics, social acceptance and environmental change. According to the tenor metrics, mobility support, community membership commitment, low-costs of resources and quality of services can guarantee social acceptance and willingness of users to modify their environment to accommodate the system. But the quality of services might not be a good measure of cost reduction as quality services are expected to be more expensive. But access to services can guarantee possible cost reduction.

\subsection{Frameworks comparison}

To evaluate our gFrame for its effectiveness in addressing the challenges of the conventional healthcare system it is compared with three other frameworks based on the tenor metrics. These systems were carefully selected from the literature based on their promises to deliver ubiquitous healthcare service provisioning. The comparative study also enables us to establish the novelty and uniqueness of our framework for ubiquitous healthcare service provisioning. These frameworks are OnkoNet (Kirn, 2002), Ubiquitous Healthcare System (UHS) (Jung et al., 2008) and Secure Ubiquitous Healthcare System Architecture (SUHSA) (Nikolidakis et al., 2010).

The OnkoNet is a mobile agents-based architecture to address the problem of accessibility to healthcare services by individual consumers using mobile devices. The OnkoNet architecture supports knowledge intensive cooperation among humans and software agents to produce, deliver, control and consume healthcare services in virtual communities supporting the cooperation of actors involved in cancer diagnosis and therapy. The OnkoNet supports local and mobile access and achieves a much better integration of patients and healthcare practitioners. While the architecture promises to support community based healthcare management that can facilitate cost reduction, it does not integrate into grids to fully support accessibility and quality of service provisioning.

The UHS consisted of vital signs devices and environment sensor devices to acquire context information to monitor and manage health status of patients anytime anywhere. The framework targeted the development of four healthcare applications including selfdiagnosis, remote monitoring, exercise management and emergency services. However, the framework is not based on virtual community and does not integrate into grids to fully provide quality and ubiquitous services at reduced costs.

The SUHSA was proposed to enable a real-time collection of healthcare data from body sensors. The collected data are converted to a CDA format, digitally signed, encrypted and securely transmitted over the Internet protocol Multimedia Subsystem (IMS) and Health Level 7 (HL7) messaging standards to a central hospital from where data can be accessed by doctors to assess the patient's health condition. The IMS provides internet services with QoS and integrates different services as well. While this architecture supports patient's mobility and security, it does not support grids and community based technologies for service provisioning. 
Table 4 shows the summary of comparing four different ubiquitous healthcare service frameworks where the rating of 0 means a column framework does not support row metric, 1 means partial support and 2 means full support. The total evaluation score received by each framework based on ability to address the challenges of conventional healthcare system shows that gFrame seems to be more promising because of its vision to integrate MDVC into the Healthgrid for ubiquitous, quality and cost-effective healthcare service provisioning. It can also be observed that OnkoNet seems to address the cost reduction challenge and SUHSA addresses the quality service challenge of the conventional healthcare system. However, OnkoNet, SUHSA and UHS frameworks do not consider all the three challenges of conventional healthcare system in the way that gFrame does, therefore making our framework unique in addressing the important challenges of healthcare system.

\begin{tabular}{|l|c|c|c|c|}
\hline Metrics/Framework & gFrame & OnkoNet & UHS & SUHSA \\
\hline Quality Services & 2 & 1 & 1 & 2 \\
\hline Access to Services & 2 & 1 & 1 & 1 \\
\hline Cost Reduction & 2 & 2 & 1 & 1 \\
\hline Total Score & 6 & 4 & 3 & 4 \\
\hline
\end{tabular}

Table 4. Frameworks comparison based on tenor metrics

\section{Conclusion}

This particular chapter discusses the roles of telemedicine, wireless body area network and wireless utility grid computing technologies to address the challenges of conventional healthcare system. We discuss the possible integration of these technologies to realize a framework for ubiquitous healthcare service provisioning. Moreover, our framework was compared with some three other ubiquitous healthcare frameworks and it promises to address the challenges of healthcare system by taking advantages of grid computing and mobile computing technologies. Our framework integrates MDVC of healthcare into the Healthgrid through a proxy based approach for performance benefits. The framework allows for remote vital signs acquisition and personalized grid service discovery through a metadata and Fuzzy logic based intelligent context engine.

Our current approach to address the challenges of the conventional healthcare system builds on innovative exploitation of the emerging technologies to realize a grid enabled framework for ubiquitous healthcare service provisioning. This framework provides a means to empower individuals in the rural world to have convenient access to quality healthcare services at reduced costs through mobile devices that they carry about without necessarily moving to the physical locations that those services are offered.

The development of a grid enabled framework integrating virtual communities of healthcare into grids is generally challenging, but it promises to yield enticing outcomes to enable ubiquitous healthcare service provisioning. In addition to addressing the challenges of healthcare system, our framework also addresses a number of research problems to support context awareness, mobility and personalized grid service discovery. The framework infrastructure is currently under development and we hope to deploy it in the future. It is also part of our future plan to consider evaluating the framework from a user perspective and to test its efficacy for enabling ubiquitous rural healthcare service provisioning. 


\section{References}

Abuelmaatti, A. \& Rezgui, Y. (2008). Virtual organizations in practice: a European perspective. Proceedings of Americas Conference on Information Systems (AMCIS).

Amendolia, S.R., Estrella, F., Frate, C.D., Galvey, J., Hassan, W., Hauer, T., Manset, D, McClatchey, R., Odeh, M., Rogulin, D., Solomonides, T. \& Warren, R. (2005). Development of a Grid-based medical imaging application. Proceedings of Healthgrid 2005, 59-69.

Barrera, M., Betancu, L., Navarro, A., Cardona, N. \& Rodrigo, V.M. (2010 ). SNR estimation for on body communications in MB OFDM ultra wide band communications. Proceedings of the Fourth European Conference on Antennas and Propagation. Barcelona, Spain. 1-5.

Berman, F., Fox, G. \& Hey, T. (2003). The Grid: past, present, future: in grid computing: making the global infrastructure a reality. John Wiley $\mathcal{E}$ Sons, 9-50.

Boccia, V., Guarracino, M.R., D'Amore, L. \& Laccetti, G. (2005). A grid-enabled PSE for medical imaging: experiences on MediGrid, In Proceedings of $18^{\text {th }}$ IEEE Symposium Comput-Based Med. Syst. (CBMS), 529-536.

Brady, M., Gavagham, D., Simpson, A., Parada, M.M. \& Highnam, R.(2003). eDiaMoND: a grid-enabled federated database of annotated mammograms. In Grid Computing: Making the Global Infrastructure a Reality, 3rd ed. New York Wiley Series, 923-943.

Breton, V., Medina, R. \& Montagnat, J. (2003). DataGrid, prototype of a biomedical grid. Methods Inf. Med. (MIM), 42, 143-148.

Chavez-Santiago, R., Khaleghi, A., Balasingham, J. \& Ramstad, T.A. (2009). Architecture of an ultra wideband wireless body area network for medical applications. Proceedings of $2^{\text {nd }}$ IEEE Intl. Symp. On Applied Sciences in Biomedical and Communication Technology (ISABEL 2009), Bratislava, Slovakia.

Chen, Y.F., Huang, H., Jana, R., Jim, T., Hiltunen, M., John, S., Jora, S., Muthumanickam, R \& Wei, B. (2003). iMobile EE - an enterprise mobile service platform. Wireless Networks, 9, 283-297.

Chu, D.C. \& Humphrey, M. (2004). Mobile OGSI.NET: grid computing on mobile devices. $5^{\text {th }}$ IEEE/ACM International Workshop on Grid Computing, 182-191.

El Morr, C. \& Kawash, J. (2007). Mobile virtual communities research: a synthesis of current trends and a look at future perspectives. International Journal of Web Based Communities, 3(4), 386-403.

Foster, I. \& Kesselmann, C. (1999). The grid: blueprint for a new computing infrastructure. Morgan Kaufmann, San Francisco.

Foster,I., Kesselman, C. \& Tuecke,S. (2001). The anatomy of the grid: enabling scalable virtual organizations. International Journal of Supercomputer Applications, 15(3), 200220.

Foster, I. (2002). What is the grid? A three checklist, GridToday, 1(6), 22-25.

Foster, I. (2006). Globus toolkit version 4: software for service-oriented systems. Journal of Comput. Sci. \& Technol., 21(4), 513-520.

Geddes, J., Lloyd, S., Simpson, A., Rossor, M., Fox, N., Hill, D., Hajnal, J.V., Lawrie, S., Mcintosh, A., Johnstone, E., Wardlaw, J., Perry, D., Procter, R., Bath, P. \& Bullmore, E. (2005). NeuroGrid: using grid technology to advance neuroscience,. In Proceedings of $18^{\text {th }}$ IEEE Symposium Comput-Based Med. Syst. (CBMS), 570-572.

Gjermundrod, H., Dikaiakos, M.D., Zeinalipour-Yazti, D., Panayi, G \& Kyprianou, T. (2007). ICGrid: enabling intensive care medical research on the EGEE grid. In from Genes 
to Personalized HealthGrid: Grid Solutions for the Life Sciences. Proceedings of Healthgrid, 248-257.

Grethe, J.S., Baru, C., Gupta, A., James, M., Ludaescher, B., Martone, M.E., Papadopoulos, P.M., Peltier, S.T., Rajasekar, A. \& Santini, S. (2005). Biomedical informatics research network: building a national collaboration to hasten the derivation of new understanding and treatment of disease. Proceedings of Healthgrid, 100-109.

Hwang, J. \& Aravamudham, P. (2004). Middleware services for P2P computing in wireless grid networks. Internet Computing, 8(4), 40-46.

Isaiadis, S., \& Getov, V. (2005). A lightweight platform for integration of mobile devices into pervasive grids. In Proceedings of High Performance Computing and Communications, Sorrento, Italy, 1058-1063.

Jacq, N., Salzemann, J., Legre, Y., Reichstadr, M., Jacq, F., Zimmermann, M., Maab, A., Srihar, K., Schnichtenberg, H., Hofmann, M. \& Breton, V. (2006). Demonstration of in silico docking at a large scale on grid infrastructure. Proceedings of HealthGrid Conference Studies in Health Technology and Informatics, 120, 155-157.

Jähnert, J.,Mandic, P.,Cuevas, A.Wesner, S. Moreno, J.I., Villagra, V., Olmedo, V. \& Stiller, B. (2010). A prototype and demonstrator of Akogrimo's architecture: an approach of merging grids, SOA and the mobile internet. Computer Communications, 33, 13041317.

Jung, J., Ha, K., Lee, J., Kim, Y. \& Kim, D. (2008). Wireless body area network in a ubiquitous healthcare system for physiological signal monitoring and health consulting. International Journal of Signal Processing, Image Processing and Pattern Recognition, $1(1), 47-54$.

Kaur, D. \& Sengupta, J. (2007). Resource discovery in web-services based grids. World Academy of Science, Engineering and Technology, 31, 284-288.

Kim, S. (2002). Ubiquitous healthcare: the OnkoNet mobile agents architecture. Proceeding Workshop on Mobile Computing in Medicine, 105-118.

Kobryn, C. \& Sibbald, C. (2004). Modeling DoDAF compliant architectures. A Telelogic White Paper.

URL: www.uml-forum.com/docs/papers/White_Paper_Modeling_DoDAF_UML2.pdf.

Latre, B., Braem, B., Moerman, I., Blondia, C. \& Demeester, P. (2010 ). A survey on wireless body area networks. Wireless Networks Manuscript.

URL: www.pats.ua.ac.be/content/publications/2010/bbraem10wbansurvey.pdf

Leimeister, J.M., Daum, M. \& Kremar, H. (2002). Mobile communication and computing in healthcare - designing and implementing mobile virtual communities for cancer patients. In Proceedings of the Tokyo Mobile Business Roundtable, Tokyo.

Leu, F. \& Wang, T (2006). A wireless grid service platform using SIP and agents. Proceedings of the Seventh ACIS International Conference on Software Engineering, Artificial Intelligence, Networking and Parallel/Distributed Computing.

Li, G., Sun, H., Gao, H., Yu, H. \& Cai, Y. (2009). A survey on wireless grids and clouds. Eight International Conference on Grid and Cooperative Computing. 261-267.

Li, H., Takahashi, T., Toyoda, M., Katayama, N., Mori, Y. \& Kohno, R., (2008). An experimental system enabling WBAN data delivery via satellite communication links. IEEE International Symposium on Wireless Communication Systems - ISWCS '08, 354-358.

Lioyd, S., Jiritka, M., Simpson, A.C., Highnam, R.P., Gavaghan, D.J., Watson, D. \&Brady, J.M. (2005). Digital mammography: a world without film? Methods of Information in Medicine, 44(2), 168-169. 
Manvi, S.S. \& Birje, M.N. (2010). A review on wireless grid computing. International Journal of Computer and Electrical Engineering. 2(3), 469-474.

Miles, S., Papay, J., Dialani, V., Luck, M., Decker, K., Payne, T. \& Moreau, L. (2003). Personalised grid service discovery. IEE Proceedings of Softw, 150(4), 252-256.

Moline, J. (1997). Virtual reality in health care: a survey. In: Riva, G. ed. Virtual reality in neuro-psycho-physiology. Amsterdam: IOS Press, 3-34.

Nam, J. (2009). A trust framework of ubiquitous healthcare with advanced Petri net model. Electronic Healthcare, 1, 122-129.

Naseer, A. \& Stergioulas, L.K. (2010). Web-services-based resource discovery model and service deployment on HealthGrids. IEEE Transactions on Information Technology in Biomedicine, 14(3), 838-845.

Nikolidakis, S.A., Georgakakis, E., Giotsas, V., Vergados, D.D. \& Douligeris, C. (2010). Secure ubiquitous healthcare system based on IMS and the HL7 standards. Proceedings of the 3rd International Conference on Pervasive Technologies Related to Assistive Environments, (PETRA'10), Samos Greece, 42.

Olugbara, O.O., Ojo, S.O. \& Adigun, M.O. (2007a). Requirements Engineering Framework for Information Utility Infrastructure for Rural e-Healthcare Service Provisioning. Information Resource Management Association (IRMA), Doctorate Symposium, Vancour, Canada, 1644-1647.

Olugbara, O.O., Adigun, M.O., Ojo, S.O. \& Mudali, P. (2007b). Utility Grid Computing and Body Area Network as Enabler for Ubiquitous Rural e-Healthcare Service Provisioning. Proceedings. of IEEE $9^{\text {th }}$ International Conference on e-Health Networking, Application and Services, 19-22 June, 2007, Taipei, Taiwan. 202-207.

Parikh, T.S. \& Lazowska, E.D. (2006). Designing an architecture for delivering mobile information services to rural developing world. International World Wide Web Conference (IW3C2), 791-800.

Peiravi, A. \& Farahi, M. (2010). Reliability of wireless body area networks used for ambulatory monitoring and healthcare. Life Science Journal, 7(2), 91-97.

Phan, T., Huang, L. \& Dulan, C. (2002). Challenge: integrating mobile wireless devices into the computational grid. MOBICOM'02, Atlanta, Georgia, USA.

Porter, C.E. (2004). A typology of virtual communities a multi-disciplinary foundation for future research. Journal of Computer-Mediated Communication, 10(1).

Rheingold, H. (2003). Mobile virtual communities. TheFeature.com Archives. URL: www.thefeaturearchives.com/topic/Culture/Mobile_Virtual_Communities.html.

Riva, G \& Gamberini, L. (2000). Virtual reality in telemedicine. Telemedicine Journal and eHealth, 6(3), 327-340.

Risk, M., Castrillo, F.P., Eijo, J.F.G., Ortega, C.S., Fernandez, M.B., Diaz, A.P., Solar, M.R. \& Pollan, R.R. (2009). CardioGrid: a framework for the analysis of cardiological signals in grid computing. Network Operations and Management Symposium (LANOMS, 2009), Punta del Else, 1-4.

Roy, S. (2003). State of the art of virtual reality therapy (VRT) in phobic disorders. Psychology Journal, 1(2), 176-183.

Ryckaert, J., Desset, C., Fort, A., Badaroslu, M., De Heyn, V., Wambacq, P., Van der Plas, G., Donnay, S., Van Poucke, B. \& Cyselinckx, B (2005). Ultra-wide-band transmitter for low-power wireless body area networks: design and evaluation. IEEE Transactions on Circuits and Systems, 52(12), 2515-2525. 
Scarlat, E. Maracine, V. \& Nica, A. (2007). Knowledge management for virtual reality applications in home rehabilitation virtual network. The Electronic Journal of Knowledge Management, 5(4), 477-486.

Scholtz, J. \& Cansolvo, S. (2004). Towards a framework for evaluating ubiquitous computing applications. Pervasive Computing, 82-88.

Schulzrinne, H. \& Wedlund, E. (2000). Application layer mobility using SIP. ACM SIGMOBILE Mobile Computing and Communication Review, 4(3), 47-57.

Shaikh, A., Memon, N., Memon, M. \& Misbahuddin, M. (2009). The role of service oriented architecture in telemedicine healthcare system. IEEE International Conference on Complex, Intelligent and Software Intensive Systems. 208-214.

Shirazi, H. \& Zahedian, A. (2009). A uniform method for evaluating the products of DoDAF architecture framework.MASAUM Journal of Computing. 1(3), 392-397.

Sneha, S. \& Varshney, U. (2006). Ubiquitous healthcare: a new frontier in e-health. Proceedings of Americas Conference on Information Systems (AMCIS).

Sriama, S.N., Jarke, M. \& Prinz, W. (2006), Mobile web service provisioning. Proceedings of the Advanced International Conference on Telecommunicat.ons and International Conference on Internet and Web Applications and Services (ICIW'06). Guadeloupe, French Caribbean.

Sun, L., Hu, P., Goh, C., Hamadicharef, B., Ifeachor, E., Barbounakis, I., Zervakis, M., Nurminen, N., Varri, A., Fontanelli, R., Di Bona, S., Guerri, D., La Manna, S., Cerbioni, K., Palanca, E. \& Starita, A. (2006). Bioprofiling over grid for eHealthcare. Proceedings of Healthgrid, Valencia Spain.

Travostino, F., Mambretti, J. \& Edwards, G.K. (2006). Grids networks: enabling grids with advanced communication technology. John Wiley.

Van Beijnum, B.J.F., Pawar, P., Dulawan, C.B. \& Hermens, H.H. (2009). Mobile virtual communities for telemedicine: research challenges and opportunities. International Journal of Computer Science and Applications, 6(2), 19-37.

Van Dam, K., Pitchers, S. \& Barnard, M. (2001). Body area networks: towards a wearable futures. In Proceedings of WWRF kick off meeting, Munich Germany.

Van Halteren, A. \& Pawar, P. (2006). Mobile service platform: a middleware for nomadic mobile service provisioning. IEEE International conference on ireless and Mobile Computing, Networking and Communication,(WIMOB2006), 292-299.

Van't Klooster, J., Pawar, P., van Beijnum, B., Dulawan, C. \& Hermens, H. (2010). Perspectives on the viable mobile virtual community for telemedicine. Encyclopedia of E-Business Development and Management in the Global Economy, 824-835.

Waldburger, M. and Stiller, B. (2005). Towards the mobile grid: service provisioning in a mobile dynamic virtual organization. Technical Report, University of Zurich, 7, 1-12.

Ylisaukko-oja, A., Vildjiounaite, E. \& Mantyjarvi, J. (2004), Five-point acceleration sensing wireless body area network - design and practical experiences. Eighth International Symposium on Wearable Computers. (ISWC2004), 1,184-185.

Yokoo, M., Durfee, E.H., Ishida, T. \& Kuwabara, K. (1998). The distributed constraint satisfaction problem: formalization and algorithms. IEEE Transactions on Knowledge and Data Engineering, 10(5), 673-685.

Zhang, D., Yu, Z. \& Chin, C. (2004). Context-aware infrastructure for personalized healthcare. The International Workshop on Personalized Health, Belfast, Northern Ireland. 


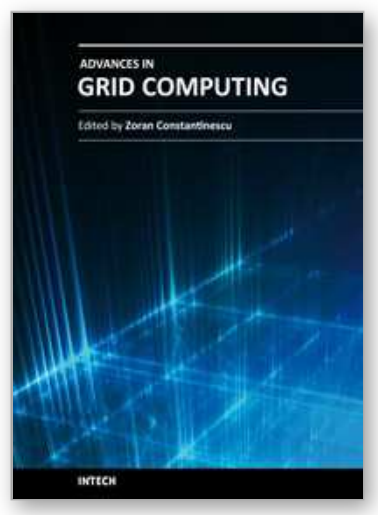

\author{
Advances in Grid Computing \\ Edited by Dr. Zoran Constantinescu
}

ISBN 978-953-307-301-9

Hard cover, 272 pages

Publisher InTech

Published online 28, February, 2011

Published in print edition February, 2011

This book approaches the grid computing with a perspective on the latest achievements in the field, providing an insight into the current research trends and advances, and presenting a large range of innovative research papers. The topics covered in this book include resource and data management, grid architectures and development, and grid-enabled applications. New ideas employing heuristic methods from swarm intelligence or genetic algorithm and quantum encryption are considered in order to explain two main aspects of grid computing: resource management and data management. The book addresses also some aspects of grid computing that regard architecture and development, and includes a diverse range of applications for grid computing, including possible human grid computing system, simulation of the fusion reaction, ubiquitous healthcare service provisioning and complex water systems.

\title{
How to reference
}

In order to correctly reference this scholarly work, feel free to copy and paste the following:

Oludayo, O., Olugbara, Sunday, O. Ojo, and Mathew, O. Adigun (2011). A Grid Enabled Framework for Ubiquitous Healthcare Service Provisioning, Advances in Grid Computing, Dr. Zoran Constantinescu (Ed.), ISBN: 978-953-307-301-9, InTech, Available from: http://www.intechopen.com/books/advances-in-gridcomputing/a-grid-enabled-framework-for-ubiquitous-healthcare-service-provisioning

\section{INTECH}

open science | open minds

\author{
InTech Europe \\ University Campus STeP Ri \\ Slavka Krautzeka 83/A \\ 51000 Rijeka, Croatia \\ Phone: +385 (51) 770447 \\ Fax: +385 (51) 686166 \\ www.intechopen.com
}

\author{
InTech China \\ Unit 405, Office Block, Hotel Equatorial Shanghai \\ No.65, Yan An Road (West), Shanghai, 200040, China \\ 中国上海市延安西路65号上海国际贵都大饭店办公楼 405 单元 \\ Phone: +86-21-62489820 \\ Fax: +86-21-62489821
}


(C) 2011 The Author(s). Licensee IntechOpen. This chapter is distributed under the terms of the Creative Commons Attribution-NonCommercialShareAlike-3.0 License, which permits use, distribution and reproduction for non-commercial purposes, provided the original is properly cited and derivative works building on this content are distributed under the same license. 